\title{
DEVELOPMENT OF A ROBUST IMAGE MOSAICKING METHOD FOR SMALL UNMANNED AERIAL VEHICLE
}

\author{
J. Kim and T. Kim* \\ Dept. of Geoinformatic Engineering, Inha University, Incheon, Korea- jikim3124@inha.edu, tezid@inha.ac.kr
}

Commission VI, WG VI/4

KEY WORDS: UAV, Image Mosaicking, Imaging Geometry Characteristics, Near-ortho mosaic

\begin{abstract}
:
In this paper, a tie-point based image mosaicking method considering imaging characteristics of small UAVs is proposed. Small UAVs can be characterized to have unstable flight trajectory and lower flight height. The proposed method considers the imaging characteristics in image transformation estimation and image blending process. For image transformation estimation, an optimal transformation model is variably applied by using tie-point area ratio. The optimal tie-point area ratio was about 0.3 . Mosaicking error was largely decreased by using this tie-point area ratio. For image blending, a composite area minimization is introduced as a preceding step of image resampling. Composite areas of individual images were minimized by analyzing image overlaps between adjacent images. The proposed method was evaluated over flat area and urban area with highly overlapping multi-strip and inconsistently overlapping strip. Experiment results showed that the proposed method can reliably generate mosaics not only from UAV images acquired in good environment but also from extreme environment.
\end{abstract}

\section{INTRODUCTION}

As the application field of small UAV increases, tie-point based mosaicking methods have been actively researched. For example, researches to solve problems caused by using a large number of images, such as mosaicking acceleration, brightness correction, and seamline extraction methods were studied (Moussa and ElSheimy, 2016; Mehrdad et al., 2016; Li et al., 2012; Bu et al., 2016). However, they did not fully consider inherent characteristics of images from small UAV. Compared to conventional platforms, the biggest difference with small UAVs is their imaging geometry characteristics such as unstable flight attitude and low flight height. These characteristics can cause various problems during image mosaicking process.

The first problem is inconsistent overlap due to unstable flight attitude. Small UAVs show high sensitivity to external environment changes and they may acquire images on unstable flight attitude.

The second problem is deformation of mosaicked image due to unstable flight attitude. In tie-point based mosaicking process, a reference image to be used as a mosaic plane has to be determined. If an image with tilt is determined as the reference image, unnecessary deformation may occur in the mosaicked image.

The third problem is larger relief displacement effect due to low altitude flight.

The fourth problem is bias of tie-point distribution due to lack of textures compared to images of conventional remote sensing platforms.

In this paper, we propose a tie-point based mosaicking method considering these imaging geometry characteristics of small UAVs. This paper focuses on geometric correction including image transformation estimation and image blending. In the transformation estimation process, transformations of individual images for mosaic image generation are established. This process can be divided into pairwise transformation estimation of adjacent images and global transformation estimation of individual images with respect to a reference plane. For a pairwise transformation estimation, we propose a method which can variably employ an optimal model according to the imaging condition. For a global transformation estimation, we propose a method which considers the imaging geometry characteristics based on a graph method. In the image blending process, individual images are transformed and composed to generate a mosaic image. To mitigate relief displacement, we propose a composite area minimization method. To evaluate the performance of the proposed method, a multi strip dataset for a flat area and a multi strip dataset for an urban area were produced. Evaluation was performed for flat area and urban area with highly overlapping images and with inconsistently overlapping images.

\section{PROPOSED METHOD}

The proposed method consists of tiepoint extraction, transformation estimation, and image blending. Figure 1 shows the procedure of the proposed image mosaicking.

In the tiepoint extraction process, tiepoint are acquired through FREAK (Fast Retina Keypoint) algorithm and RANSAC (RANdom SAmple Consensus) algorithm (Alexandre et al., 2012; Fischler and Bolles, 1981). This process generally requires a large amount of computation in feature matching. In order to mitigate this problem, an adjacent image pair detection step that can minimize unnecessary computation by determining matching image pairs in advance is introduced.

In the image transformation estimation process, pairwise transformations between adjacent images are estimated and global transformations of individual images for a reference plane are derived from the estimated pairwise transformations. For the

\footnotetext{
* Corresponding author
} 


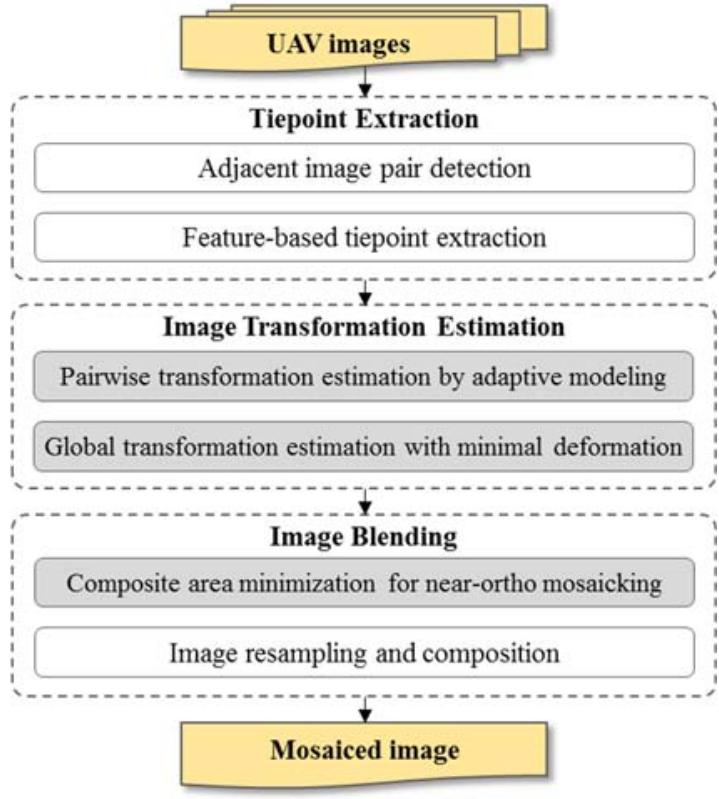

Figure 1. Work flow for the proposed method

pairwise transformation estimation, a method which can variably employ an optimal model according to the imaging condition is applied. The proposed method measures estimation confidences for adjacent images, and estimates the pairwise transformations by variably applying a precision model and a simple model according to a confidence criterion. This approach aims at securing high accuracy by applying a precision model when error factors are small, and securing high estimation stability by applying a simple model when error factors are large. For confidence criterion, tie-point area ratio (TAR) has been introduced. The TAR is defined as the ratio of the tiepoint area to the entire image area. We found that the accuracy of the precision model and simple model was reversed at TAR value of 0.3 by analyzing correlation between transformation accuracy and TAR according to image overlap change. Accordingly, we chose TAR 0.3 as the model selection criterion. Figure 2 shows the result of the correlation analysis.

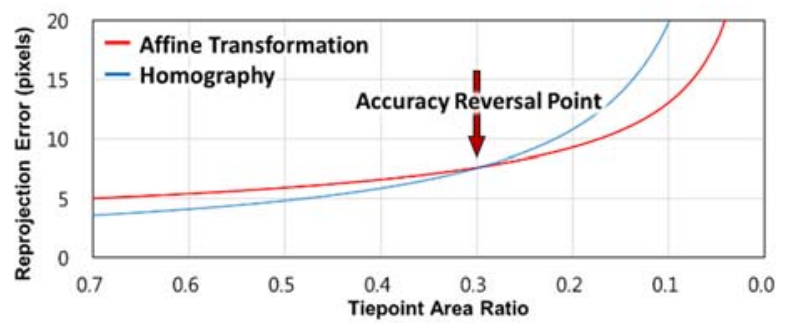

Figure 2. Comparison of correlation models established between transformation accuracy and tiepoint area ratio.

For the global transformation estimation, a method which can consider the imaging geometry characteristics based on a graph method is applied. The graph method is divided into maximum spanning tree (MST) generation and reference plane determination phase (Marzotto et al., 2004; Kim and Kim, 2017). In MST generation, the proposed method applies TAR as weight to consider bias of tiepoint distribution. In reference plane determination, the proposed method defines an image that minimizes the mosaicking deformation as a reference plane to prevent unnecessary deformation.
In the image blending process, a composite area minimization is introduced before image resampling and image composition step. A composite area is an image region actually used for image mosaicking. Therefore, composite area minimization means to reduce composite areas of individual images based on their image center unless holes are occurred in the mosaicking result. Since this approach uses only image central region, it is possible not only to generate near-ortho image mosaic but also to improve the processing efficiency. Figure 3 shows the procedure of the proposed method.

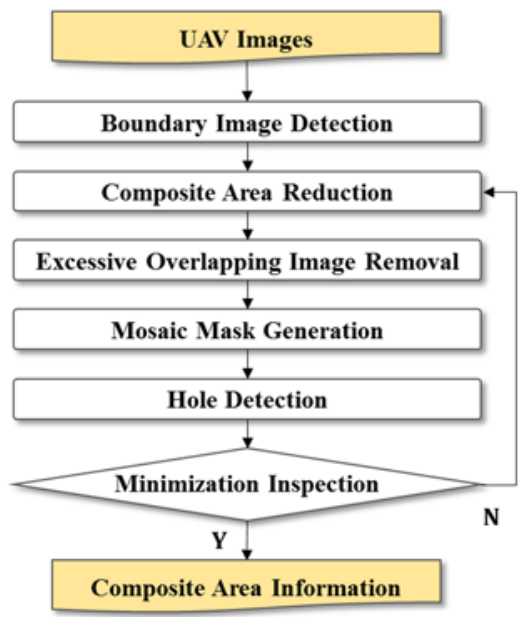

Figure 3. Work flow for composite area minimization

In the boundary image detection step, images are divided into boundary images and internal images for an image mosaic. This is to restrict information loss in the outer part of the image mosaic by reducing the composite areas of the boundary images and the internal images differently.

In the composite area reduction step, composite areas of images are reduced to a certain size in the direction of their image center by adjusting the angle-of-view. In this step, internal images are continuously reduced until the minimization is achieved. Boundary images are reduced only until a certain threshold value predetermined for angle-of-view.

In the excessive overlapping image removal step, when the inclusion relation is established between the adjacent images due to the composite area reduction, the images included in the other images are removed.

In the mosaic mask generation step, a mask image for a mosaicking result is generated. This facilitates hole detection in the next step.

In the hole detection step, holes to be occurred inside image mosaic are detected by analyzing the mosaic mask. When holes are detected in the current processing loop, the composite areas of the images involved in the holes are restored to those of the previous processing loop, and then the composite areas of the images are fixed.

Finally, in the minimization inspection step, it is determined whether to repeat the composite area reduction process. If there are images without composite area minimization, the processing step returns to the composite area reduction step and repeats the same process. 


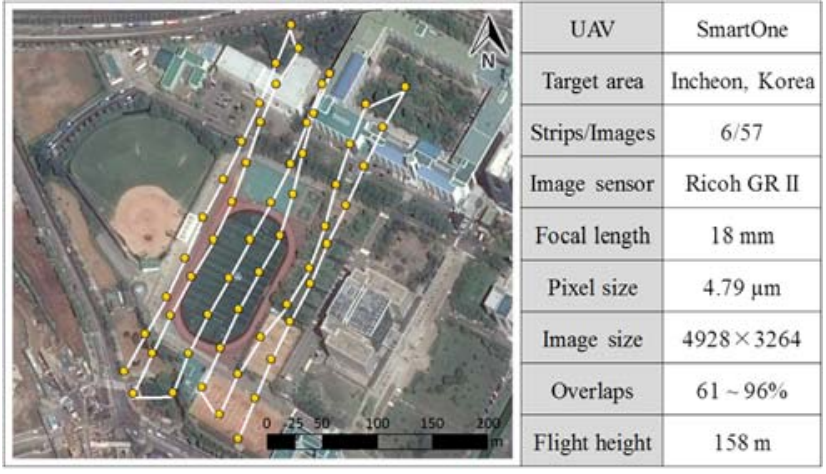

(a) Dataset-2

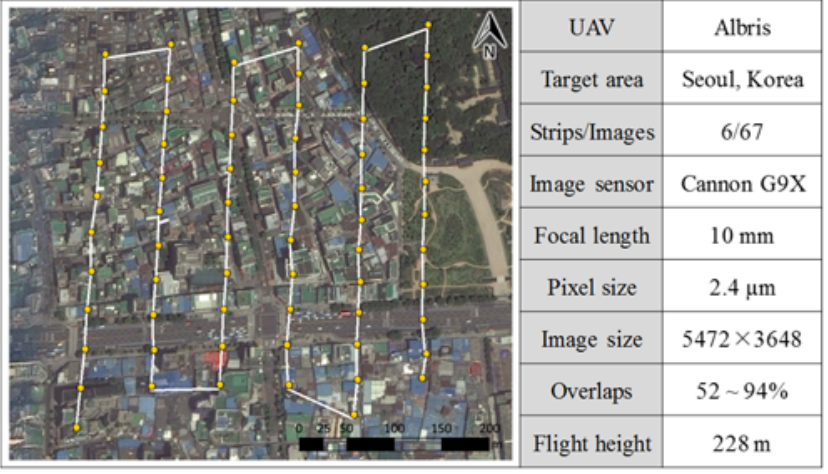

(b) Dataset-3

Figure 4. Image datasets produced for evaluation of the proposed method

\section{RESULT AND DISCUSSION}

The proposed method was evaluated by two experiments. In the first experiment, we evaluated the mosaicking performance for low altitude flight using highly overlapping image strips. In the second experiment, we evaluated the mosaicking performance for unstable flight attitude using inconsistently overlapping image strips.

For reliable evaluation, performance comparison with existing mosaicking method was carried out. In the existing method, a single transformation model was applied equally for pairwise transformation estimation, and a graph based method was applied for global transformation estimation. For image blending, the image resampling and image composition were only performed.

The image mosaics were produced by dividing each of them according to transformation models used to estimate pairwise transformations. For the existing method, affine transformation model and homography model were used. For the proposed method, a hybrid model of affine transformation and homography was used. In the evaluation result, they were labeled Affine Transform, Homography, and Hybrid Transform.

\subsection{Datasets}

For the evaluation, multi-strip image datasets were produced for flat and urban areas using small UAVs. The flat area images (Dataset-2) include some buildings in the outer part of the strip but generally show gentle terrain variations. On the other hand, the urban area images (Dataset-3) show complicated terrain variations including high and low features throughout the strip. Detailed specifications for each of the Dataset- 2 and -3 can be seen in Figure 4.

\subsection{Evaluation for highly overlapping images}

Transformation errors and deformations were measured to analyse the mosaicking accuracy. The transformation errors were calculated as the reprojection error for the entire image mosaic. The image distortions were calculated as the root mean square error for the orthogonality difference between the original images and the transformed images. Table 1 shows the evaluation results for the transformation estimation.

The results in Table 1 showed that the proposed method had higher performance than the exist methods. These results were the same for both the flat area images and urban area images. In the results, the average TAR values were greater than 0.3 . This implies that most of the transformations were estimated using a precision model in pairwise transformation estimation process. Therefore, it can be interpreted that this improvement was mainly due to the proposed global transformation estimation method. These results demonstrate that the proposed method for global transformation estimation is effective in mitigating the problems caused by low altitude flight and unstable flight attitude.

In the image blending process, composite areas of images were minimized before image resampling. To analyse the performance improvement due to the composite area minimization, result comparison was carried out as shown in Table 2.

The results showed that the transformation estimation accuracy was improved by about 2 times through the composite area minimization. Compared with the results of existing method using a precision model, the improvements were about 6 times and 3 times for the Dataset- 2 and -3 , respectively. This is because the geometric inconsistency can be reduced by using only the image central regions close to the ortho projection in image mosaic generation.

In order to confirm the improvement in more detail, visual analysis was performed. Among the existing methods, the method using a precision model showed relatively better performance. Figure 5 shows the mosaicking results of the proposed method and the existing method.

The quantitative analysis results were also confirmed in the image mosaics generated. In the results of Homography, geometric inconsistencies were largely observed (red ellipses), whereas in the results of Hybrid Transform-A, the misalignments were greatly alleviated.

The results of Hybrid Transform-B showed that it is possible to generate near-ortho image mosaics by applying composite area minimization. In the results of Hybrid Transform-A without applying composite area minimization, the problems due to relief displacement were observed (yellow ellipses), whereas the results of Hybrid Transform-B showed that these problems were greatly alleviated. These results show that the proposed method can be effectively used to mitigate the relief displacement problems caused by low altitude flight.

\subsection{Evaluation for inconsistently overlapping images}

In the evaluation of Section 3.2, highly overlapping multi-strip images were used. For this reason, as most of the image pairs selected for global transformation estimation forms high TAR, the evaluation of the proposed method was limited to the global 
The International Archives of the Photogrammetry, Remote Sensing and Spatial Information Sciences, Volume XLII-2/W6, 2017 International Conference on Unmanned Aerial Vehicles in Geomatics, 4-7 September 2017, Bonn, Germany

Table 1. Transformation estimation accuracy for Dataset-2 and -3.

\begin{tabular}{|c|c|c|c|c|c|c|c|c|c|c|}
\hline \multirow{3}{*}{ Modeling Method } & \multicolumn{5}{|c|}{ Dataset-2 } & \multicolumn{5}{|c|}{ Dataset-3 } \\
\hline & \multicolumn{3}{|c|}{ Tiepoint Area Ratio } & \multirow{2}{*}{$\begin{array}{l}\text { Reprojection } \\
\text { Error(pixels) }\end{array}$} & \multirow{2}{*}{$\begin{array}{c}\text { Deformation } \\
\text { (Degree) }\end{array}$} & \multicolumn{3}{|c|}{ Tiepoint Area Ratio } & \multirow{2}{*}{$\begin{array}{l}\text { Reprojection } \\
\text { Error(pixels) }\end{array}$} & \multirow{2}{*}{$\begin{array}{l}\text { Deformation } \\
\text { (Degree) }\end{array}$} \\
\hline & Min. & Mean & Max. & & & Min. & Mean & Max. & & \\
\hline Affine Transform & 0.32 & 0.56 & 0.85 & 475.60 & 5.18 & 0.24 & 0.50 & 0.73 & 362.08 & 3.49 \\
\hline Homography-LM & 0.32 & 0.56 & 0.85 & 59.88 & 7.09 & 0.24 & 0.49 & 0.73 & 149.33 & 6.30 \\
\hline Hybrid Transform & 0.38 & 0.60 & 0.85 & 18.78 & 3.87 & 0.33 & 0.51 & 0.73 & 59.54 & 4.52 \\
\hline
\end{tabular}

Table 2. Comparison of transformation estimation accuracy by applying composite area minimization.

\begin{tabular}{c|c|c|c|c|c|c|c}
\hline \multirow{2}{*}{ Modeling Method } & \multirow{2}{*}{$\begin{array}{c}\text { Composite } \\
\text { Area } \\
\text { Minimization }\end{array}$} & $\begin{array}{c}\text { No. of } \\
\text { Images }\end{array}$ & Min. AOV & $\begin{array}{c}\text { Reprojection } \\
\text { Error (pixels) }\end{array}$ & $\begin{array}{c}\text { No. of } \\
\text { Images }\end{array}$ & $\begin{array}{c}\text { Min. } \\
\text { AOV }\end{array}$ & $\begin{array}{c}\text { Reprojection } \\
\text { Error (pixels) }\end{array}$ \\
\hline Hybrid Transform-A & $\mathrm{N}$ & 57 & 46.95 & $\mathbf{1 8 . 7 8}$ & 67 & 46.67 & $\mathbf{5 9 . 5 4}$ \\
Hybrid Transform-B & $\mathrm{Y}$ & 39 & 2.95 & $\mathbf{9 . 4 6}$ & 51 & 8.27 & 31.12 \\
\hline
\end{tabular}

Table 3. Mosaic deformation results for the subsets of Dataset-2 and -3

\begin{tabular}{|c|c|c|c|c|c|c|c|c|}
\hline \multirow{3}{*}{ Modeling Method } & \multicolumn{4}{|c|}{ Dataset-2 } & \multicolumn{4}{|c|}{ Dataset-3 } \\
\hline & \multicolumn{3}{|c|}{ Tiepoint Area Ratio } & \multirow{2}{*}{$\begin{array}{c}\text { Deformation } \\
\text { (Degree) }\end{array}$} & \multicolumn{3}{|c|}{ Tiepoint Area Ratio } & \multirow{2}{*}{$\begin{array}{c}\text { Deformation } \\
\text { (Degree) }\end{array}$} \\
\hline & Min & Mean & Max & & Min & Mean & Max & \\
\hline Affine Transform & 0.01 & 0.22 & 0.48 & 5.44 & 0.03 & 0.16 & 0.40 & 4.15 \\
\hline Homography & 0.01 & 0.22 & 0.48 & 21.23 & 0.03 & 0.16 & 0.40 & 11.97 \\
\hline Hybrid Transform & 0.01 & 0.25 & 0.48 & 4.86 & 0.03 & 0.16 & 0.40 & 2.49 \\
\hline
\end{tabular}
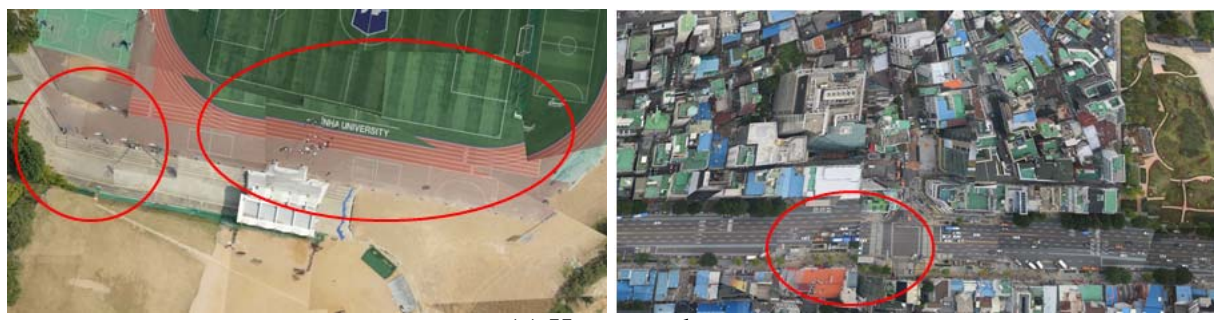

(a) Homography
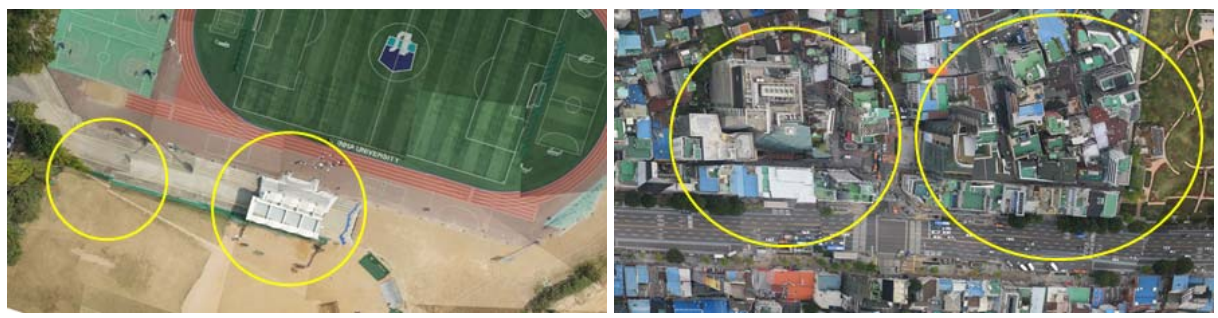

(b) Hybrid Transform-A
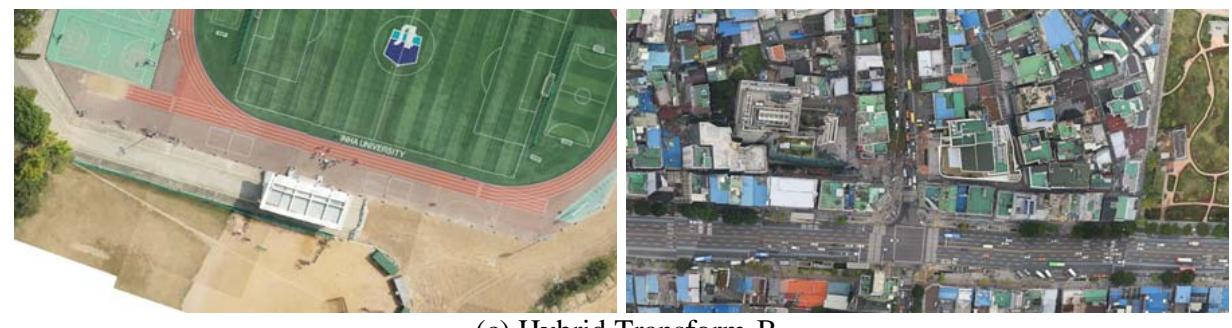

(c) Hybrid Transform-B

Figure 5. Mosaicking result comparison for the Dataset-2 (left column) and 3 (right column)

transformation estimation method and the composite area minimization method. In this section, the proposed method for pairwise transformation estimation was further analyzed using inconsistently overlapping images constructed from Dataset-2 and -3 . This image data setting simulates extreme environment for image acquisition.

In this experiment, the evaluation was performed in terms of image deformation. The image distortion was calculated from the orthogonality difference with the mosaicking result produced in the previous experiment. As a reference mosaicking result, the result of the proposed method with best performance in the previous experiment was used. Table 3 describes the evaluation results for the subsets of Dataset-2 and -3. Figure 6 shows image mosaics generated from the subsets of Dataset-2 and -3 .

In the result of Table 4, it was confirmed that large distortions were occurred in the cases applying the precision model. These 

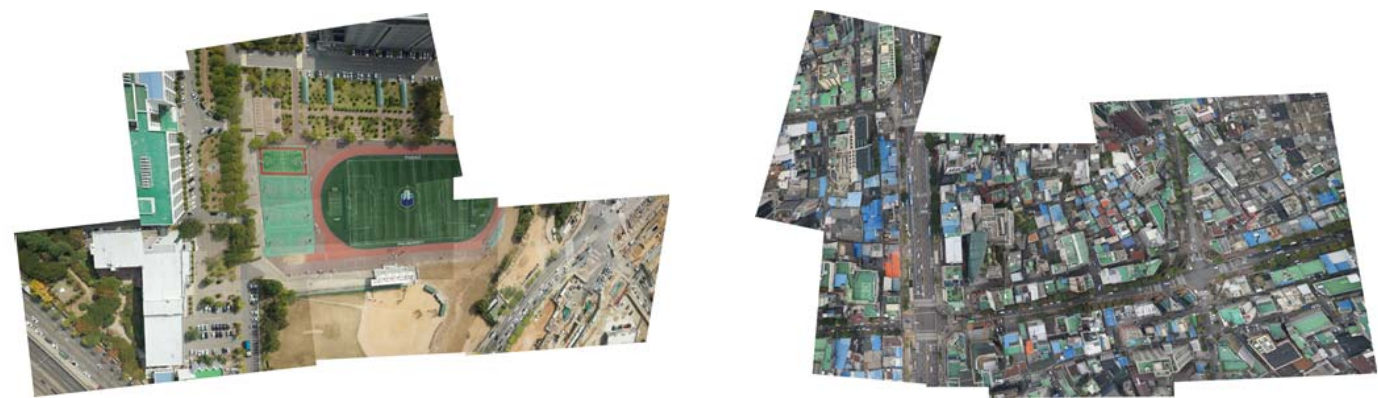

(a) Affine Transform
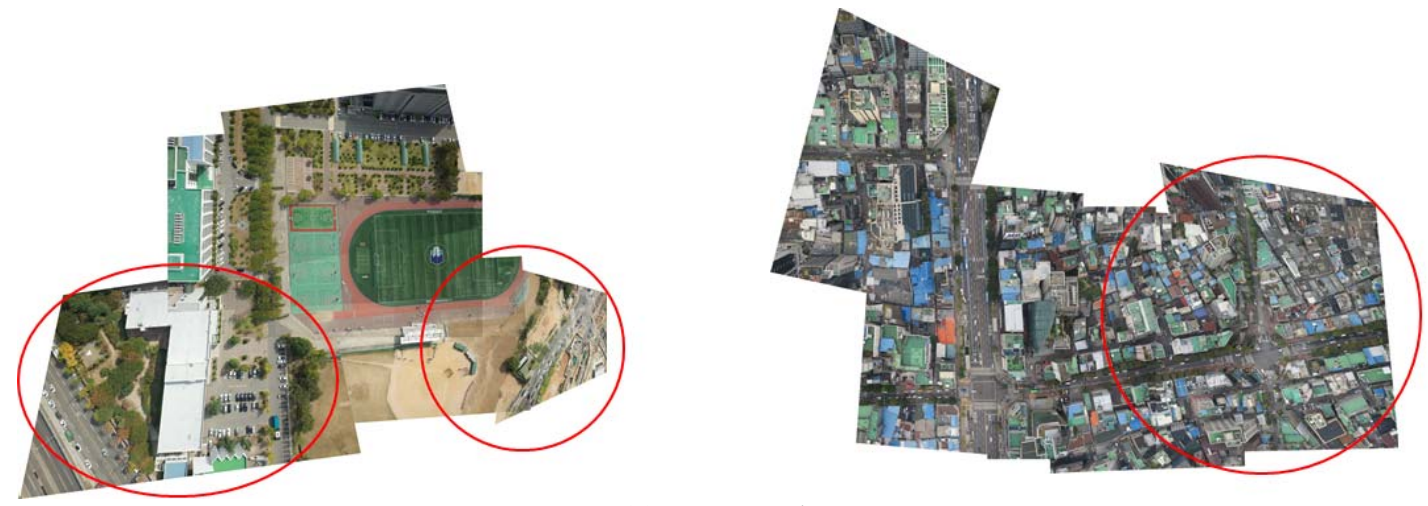

(c) Homography
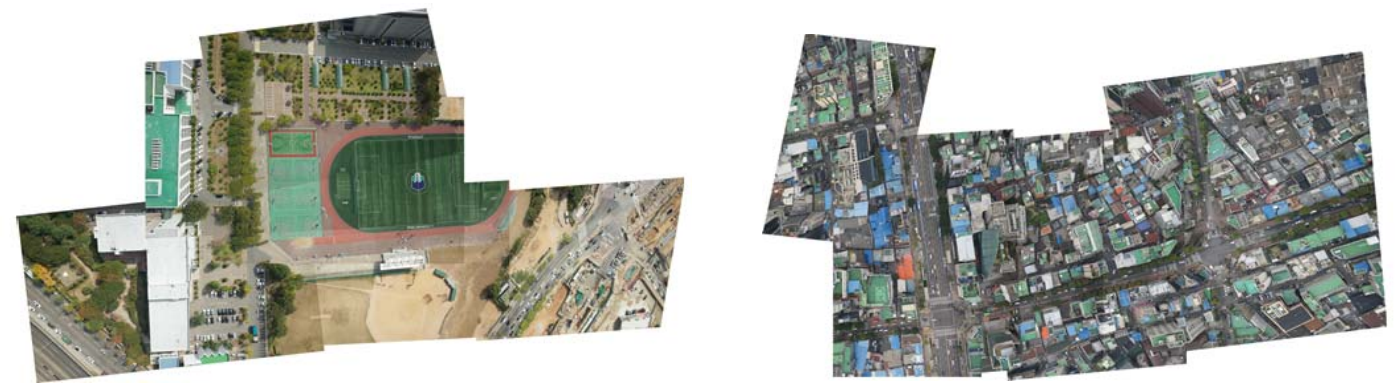

(e) Hybrid Transform

Figure 6. Mosaicking results for the subset of Dataset-2 and -3 (left column) and 3 (right column)

distortions were also confirmed in the mosaicking results of Homography in Figure 6. These results show that applying a simple transformation model rather than a precision transformation model can produce better results for images with large error factors.

On the other hand, the proposed method showed the best performance among all methods. This is because the proposed method is able to variably apply a precision model and a simple model according to the error factors between adjacent images for the image datasets forming inconsistent overlaps. These results show that the proposed method can robustly generate image mosaics even in extreme situations where image overlaps can be formed inconsistently.

\section{CONCLUSION}

In this paper, a tiepoint based image mosaicking method considering the imaging characteristics of small UAV was proposed. For image transformation estimation, we proposed a pairwise transformation estimation method and a global transformation estimation method. For a pairwise transformation estimation, we proposed a method which can variably employ an optimal model according to an estimation confidence criterion.
For confidence criterion, TAR to consider both image overlap and tiepoint distribution was proposed. The value of TAR for model selection was about 0.3. For a global transformation estimation, we proposed a graph based method which can minimize mosaicking error and distortion by using TAR and image orthogonality. The experimental results showed that the proposed method can reliably estimate image transformations not only for highly overlapping images but also for inconsistently overlapping images.

For image blending, we proposed a composite area minimization method. To mitigate relief displacement, this method minimizes composite areas of individual images by analyzing overlapping regions between adjacent images. The experimental results showed that the proposed method can generate near-ortho mosaics even in the urban area where the terrain variation is complex.

The contribution of this paper is that it has developed a reliable method for image mosaic generation not only for UAV images obtained in stable environments but also for UAV images acquire in extreme environments. 


\section{REFERENCES}

Moussa, A., and El-Sheimy, N., 2016. A fast approach for stitching of aerial Images, International Archives of the Photogrammetry, Remote Sensing and Spatial Information Sciences, Volume XLI-B3, pp.769-774.

Mehrdad, S., Satari, M., Safdary, M., Moallem, P., 2016. Toward real time UAVS' image mosaicking, International Archives of the Photogrammetry, Remote Sensing and Spatial Information Sciences, XLI-B1, pp.941-946.

Li, M., Li, D., and Fan, D., 2012. A study on automatic UAV image mosaic method for paroxysmal disaster, International Archives of the Photogrammetry, Remote Sensing and Spatial Information Sciences, Volume XXXIX-B6, pp.123-128.

Bu, S., Zhao, Y., Wan, Gang., and Liu, Z., 2016. Map2DFusion: real-time incremental UAV image mosaicing based on monocular SLAM, In: IEEE/RSJ International Conference on Intelligent Robots and Systems, pp.4564-4571.

Alexandre, A., Ortiz, R., and Vandergheynst, P., 2012. Freak: Fast retina keypoint, In: IEEE Conference on Computer Vision and Pattern Recognition, pp. 510-517.

Fischler, M.A., and R.C. Bolles. 1981. Random Sample Consensus: A Paradigm for Model Fitting with Applications to Image Analysis and Automated Cartography, Communications of the ACM, 24(6): 381-395.

Marzotto, R., Fusiello, A, and Murino, V., 2004. High resolution video mosaicing with global alignment, In: IEEE Computer Society Conference on Computer Vision and Pattern Recognition, Vol.1, pp.692-698.

Kim, J., and Kim, T., 2017. Fast and robust geometric correction for mosaicking UAV images with narrow overlaps, International Journal of Remote Sensing, 38(8-10):2557-2576. 